\title{
Impact of Hemodialysis Therapy on Anemia of Chronic Kidney Disease: The Potential Mechanisms
}

\author{
Sudhir K. Bowry ${ }^{a}$ Emanuele Gatti ${ }^{a}$ b \\ ${ }^{a}$ Fresenius Medical Care, Bad Homburg, Germany; ${ }^{b}$ Centre for Biomedical Technology at the Danube \\ University of Krems, Krems, Austria
}

\section{Key Words}

Online hemodiafiltration - Anemia of chronic kidney

disease $\cdot$ Uremic toxins

\begin{abstract}
A significant and increasing number of chronic kidney disease (CKD) patients are treated with online hemodiafiltration (OL-HDF), even in the absence of more conclusive survival data. OL-HDF affords several clinical benefits including control of anemia of CKD, a common affliction in dialysis patients. In efforts to understand the underlying mechanisms that contribute to the purported benefits of OL-HDF, we examined the potential role and impact of OL-HDF on key stages of anemia and its correction: erythropoiesis of bone marrow, circulating erythrocytes and on anemia therapy. We review evidence that indicates OL-HDF may modulate key processes of anemia and its therapy, including underlying conditions and responses of uremic toxicity and inflammation that aggravate anemia. Our assessment indicates that OL-HDF favorably impacts anemia by not only eliminating putative uremic inhibitors that suppress erythropoiesis, reducing red cell destruction and increasing iron availability, but also by mechanisms restricting underlying inflammation and endothelial dysfunction that are crucial to both CKD and anemia.

Copyright $\odot 2011$ S. Karger AG, Basel
\end{abstract}

\section{Introduction}

High mortality rates amongst chronic kidney disease (CKD) patients on hemodialysis (HD) therapies have been recognized for some time. A number of strategies to improve patient outcomes have been considered, including refinement of clinical prescription and dialysis technology on which dialysis therapy is so dependent. In particular, considerable attention has been placed in recent years on treatment duration, frequency and modalities in attempts to not only overcome the effects of uremia but also of a multitude of related comorbid conditions accompanying chronic CKD [1].

Efficient elimination of a broad spectrum of accumulated uremic retention solutes ('uremic toxins'), small and large, constitutes the core objective of all HD therapies [2]. As uremic toxicity begins to be better understood, the significance of removal of larger compounds, classified as middle molecules (MM) and known for some time to play a role in the uremic syndrome, has been realized [3]. Advances in membrane and machine technology have further facilitated the establishment of high-flux dialysis (HF-HD) as the predominant form of HD treatment [4]. Worldwide, over half of all HD patients are today treated with high-flux membranes [5]. This transition from lowflux dialysis (LF-HD) to HF-HD is an assertion of the

\section{KARGER}

Fax +4161306 1234 E-Mail karger@karger.ch www.karger.com
Sudhir K. Bowry, PhD

Scientific Affairs, Fresenius Medical Care

Else-Kröner-Strasse 1

DE-61352 Bad Homburg (Germany)

Tel. +496172 608 2128, E-Mail Sudhir.Bowry@fmc-ag.com 
clinical need to target removal of large compounds that accumulate in uremia. An open, prospective, randomized controlled trial (RCT) involving some 738 patients in 59 centers from 9 European countries demonstrated a survival benefit for HF-HD compared to LF-HD particularly in a dialysis population with increased risk of morbidity and mortality (patients with low serum levels of albumin and for diabetics) [6].

In recent years, there has been a further steady shift towards online hemodiafiltration (OL-HDF), a derivative of HF-HD that relies predominantly on the mechanism of convective solute transport across HF membranes for the enhanced removal larger substances [7-9]. In Northern Europe for example, $24 \%$ of HD patients receive OLHDF, while in Switzerland as many as $61 \%$ of patients are routinely treated with OL-HDF [unpubl. data]. While the superiority of OL-HDF in terms of efficient removal of larger toxins has been well validated and is seldom questioned, absence of more definitive clinical evidence in the form of properly designed and adequately powered prospective RCT demonstrating better survival rates is often the only deterrent to an even broader prescription of OLHDF [10]. Evidence attributing improved patient outcomes to OL-HDF is based so far on prospective observational, epidemiological, retrospective analysis, or on small (single-center) investigations [10-12]. A prospective RCT designed specifically to compare OL-HDF with standard LF-HD with respect to cardiovascular morbidity and mortality has just been concluded; the findings of the CONTRAST study are expected to help resolve perhaps the main controversy that impedes more patients from benefitting from the reported advantages of OLHDF [13]. Proponents of OL-HDF who have successfully implemented the treatment modality for over a decade in routine clinical practice in several countries emphasize the imperative of patient well-being and reduced morbidity with OL-HDF $[7,8,14]$. Improved quality of life with OL-HDF is attributed to the combination of better control of anemia, reduction in calcium-phosphate product, improved dialysis dose and more efficient removal of $\mathrm{MM}$, better control of hypertension and hemodynamic stability as well as to mechanisms involving reduced inflammatory responses, oxidative stress and endothelial dysfunction $[8,15]$. Thus, OL-HDF is used to treat a significant and increasing population of CKD patients; even in the absence of more conclusive survival data based on RCT, the modality must nevertheless afford some clinical benefits for its continued prescription.

Anemia of CKD is a major complication that occurs early in the development of $\mathrm{CKD}$, with severity increasing as renal function deteriorates [16]. Most of the patients with stage V CKD and requiring renal replacement therapies (RRT) in the form of dialysis have anemia, which contributes to poor quality of life and outcomes. Anemia of CKD is attributed primarily to insufficient nephric output of erythropoietin (EPO) and unavailability of iron leading to decreased erythropoiesis. Its correction, essentially by pharmaceutical intervention with erythropoiesis-stimulating agents (ESA) and intravenous (i.v.) iron supplements, is an important component of CKD management but adds significantly to the high overall costs of treating patients with advanced CKD [17]. Treatment of anemia of CKD has become a highly contentious topic, with growing concerns regarding the precise targets, safety, clinical evidence, questionable benefits and costs of present anemia management practices [18]. There are increased calls for a re-evaluation of anemia management strategies in HD and for alternative approaches to help correct the disorder [19]. While exogenous supplementation of EPO and iron remains the mainstay of anemia therapy in $\mathrm{CKD}$, the potential for improving the condition by curbing underlying conditions that contribute to the pathogenesis of anemia, or by dialysis-specific approaches, have yet to be fully recognized.

In efforts to understand the underlying mechanisms that may contribute to the purported decrease in mortality and morbidity with certain variants of dialysis, in this paper we examine the potential role and impact of dialysis procedures, particularly OL-HDF, on key stages of anemia and its correction: erythropoiesis of bone marrow, circulating red blood cells (RBC) and on anemia therapy itself. We review evidence that suggests dialysis therapy (treatment modality and its components) may modulate and affect key processes of anemia and its therapy, including the underlying conditions and responses of uremic toxicity, inflammation, oxidative stress and endothelial dysfunction all of which are known to aggravate anemia of CKD.

\section{Impact of Dialysis on Bone Marrow Erythropoiesis}

The key stages of RBC differentiation and maturation occur within the bone marrow. Although deficient production of EPO and unavailability of iron are the main causes of suppression of these processes, several factors contribute to decreased erythropoiesis (fig. 1). Dialysis therapy may directly affect bone marrow erythropoiesis by either removing substances that inhibit erythropoiesis, or by enhancing availability of iron. 


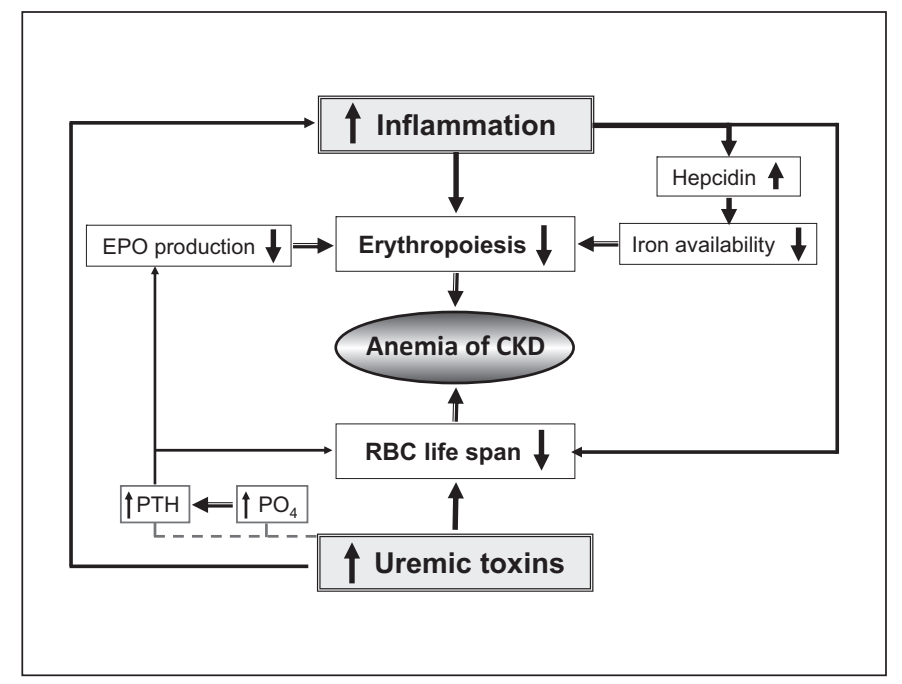

Fig. 1. Uremic toxicity and inflammation both exacerbate anemia of CKD, affecting bone marrow erythropoiesis and erythrocyte survival. Dialysis therapies that curb the effects of both uremic toxins and inflammatory mediators enable anemia correction.

\section{Removal of Putative Inhibitors of Erythropoiesis} during Dialysis

Background: Uremic Toxins Exacerbate Anemia

Evidence for the presence of uremic substances that suppress erythropoiesis was suspected when severe anemia was observed in CKD patients even at elevated levels of EPO and by the dose-dependent inhibition of bone marrow cells in culture when exposed to uremic serum [20]. The inhibitor theory gained further ground after it was shown that anemia improves after initiation of dialysis [21]. Thus, although anemia is primarily an endocrine deficiency, hypoproliferation of red cell progenitors in CKD may partially be caused by an inhibitory effect of accumulated uremic toxins. Despite the early evidence for putative inhibitors of erythropoiesis, the exact identity and role of endogenous uremic substances that inhibit erythropoiesis has been controversial and remains rather obscure. The involvement of large-sized inhibitors of anemia was first implicated by the less severe anemia observed in peritoneal dialysis patients and attributed to a more efficient removal of MM by the more porous peritoneal membrane [22]. As inhibitors of erythropoiesis are believed to be mostly large-molecular-weight substances, both HF membranes and convective therapies would thus facilitate their removal [23]. Indeed, studies have shown that removal of these high-molecular-weight substances by more permeable dialysis membranes improves the anemic state of dialysis patients [24].
In a randomized clinical study designed specifically to examine the effects of removal of inhibitors of erythropoiesis on anemia and EPO requirements, HF-HD was compared with LF-HD over 6 months in 48 patients who could not reach target hemoglobin $(\mathrm{Hb})$ levels $(\geq 11$ $\mathrm{g} / \mathrm{dl}$ ) despite treatment with subcutaneous EPO [25]. Although EPO doses were significantly lower in the HFHD group, Hb levels showed a significant increase, unlike the LF-HD group despite higher EPO doses. As there was no difference between the two groups in terms of dose of dialysis, and as only the HF group showed enhanced removal of $\beta_{2}$-microglobulin (a surrogate of MM removal), the data suggest that improved removal of unspecified, large-sized inhibitors of erythropoiesis may help correct anemia of CKD. The beneficial effects observed for HF-HD should also be apparent for OLHDF, a more efficient HD modality for the removal of larger uremic compounds. Compared to standard dialysis, OL-HDF has been shown to improve the anemic status of CKD patients by significantly increasing $\mathrm{Hb}$ to target levels at reduced maintenance doses of EPO [26]. Two further studies showed that when patients are switched from conventional HD to OL-HDF, significantly higher hematocrit (Hct) and $\mathrm{Hb}$ levels were reached with the OL-HDF treatment modality $[27,28]$ (table 1). Attainment of higher Hct and $\mathrm{Hb}$ levels with more efficient dialysis therapies (HF membranes and convective therapies) has been attributed to the elimination of larger uremic retention substances that impair erythropoiesis $[21,26]$.

Of the many compounds that have been implicated as potential inhibitors of bone marrow erythropoiesis, the most extensively investigated substances include polyamines, polyaminated peptides and parathyroid hormone (PTH) $[21,29,30]$. PTH is considered as a major MM uremic toxin with multiple biological effects, including causing anemia by a direct inhibitory effect on endogenous EPO synthesis, bone marrow erythroid progenitors (suppressing erythropoiesis) and RBC survival [31]. PTH also affects the response to exogenous ESA in HD patients; secondary hyperparathyroidism has been identified to increase EPO resistance [3, 32]. PTH levels, together with markers related to anemia correction, inflammation, oxidative stress and hyperphosphatemia, were compared for OL-HDF and LF-HD in a crossover study with 70 patients [33]. A higher elimination of PTH during OL-HDF therapy corresponded with higher Hct and $\mathrm{Hb}$ levels at lower EPO doses.

Hyperphosphatemia (highly prevalent in HD patients), by decreasing calcitriol synthesis and reducing 
Table 1. Studies examining or reporting the direct influence of dialysis therapy (treatment modality, conditions or components) on various aspects of anemia of CKD

\begin{tabular}{|c|c|c|c|}
\hline Reference & Study design/features & Patients & Observed anemia-related effects attributed to HD therapy \\
\hline Panichi et al. [68] & $\begin{array}{l}\text { Prospective, observational, prevalent patients, 36-month } \\
\text { follow-up }\end{array}$ & 753 & Decreased EPO resistance \\
\hline Pedrini et al. [69] & $\begin{array}{l}\text { Prospective, multicenter, randomized crossover, high- } \\
\text { efficiency OL-HDF versus LF-HD }(2 \times 6 \text { months })\end{array}$ & 69 & $\begin{array}{l}\text { No Hb change; reduction in maintenance doses of EPO } \\
\text { Lower inflammation } \\
\text { Better toxin removal (lower PTH and } \mathrm{Ca}-\mathrm{Po}_{4} \text { product/ } \\
\left.\beta_{2} \mathrm{~m}\right)\end{array}$ \\
\hline Malyszko et al. [41] & $\begin{array}{l}\text { Cross-sectional, assess hepcidin in relation to type of } \\
\text { RRT, renal function, iron status, markers of } \\
\text { inflammation }\end{array}$ & 185 & Enhanced removal of hepcidin by HDF \\
\hline Schiffl [66] & $\begin{array}{l}\text { Prospective, randomized crossover, switch of } \\
\text { conventional LF-HD to ultrapure HF-HD and OL-HDF; } \\
24 \text { months }\end{array}$ & 76 & $\begin{array}{l}\text { Reduction in EPO doses at target } \mathrm{Hb} \text { levels } \\
\text { Higher MM removal } \\
\text { Reduction in microinflammation }\end{array}$ \\
\hline Go et al. [60] & Prospective, switch from standard to ultrapure dialysate & 61 & $\begin{array}{l}\text { Increase in } \mathrm{Hb}, \mathrm{RBC} \text { count and Hct after switch } \\
\text { Increase in } \mathrm{RBC} \text { life span and reticulocyte count }\end{array}$ \\
\hline Carracedo et al. [54] & $\begin{array}{l}\text { Prospective, crossover, HF-HD versus OL-HDF, } \\
4 \text { months each }\end{array}$ & 31 & $\begin{array}{l}\text { Reduction in proinflammatory markers and ferritin with } \\
\text { OL-HDF }\end{array}$ \\
\hline Canaud et al. [11] & $\begin{array}{l}\text { Observational, LF-HD, HF-HD, low-/high-efficiency } \\
\text { OL-HDF }\end{array}$ & 2,165 & $\begin{array}{l}\text { Lower EPO doses with high-efficiency OL-HDF (+ higher } \\
\text { survival) }\end{array}$ \\
\hline Vaslaki et al. [33] & $\begin{array}{l}\text { Controlled, randomized crossover LF-HD to OL-HDF, } \\
24 \text { weeks }\end{array}$ & 70 & $\begin{array}{l}\text { Higher Hct } \\
\text { Reduction in EPO doses } \\
\text { Reduced inflammation }\end{array}$ \\
\hline Ayli et al. [25] & $\begin{array}{l}\text { Randomized, switch from HF-HD versus LF-HD over } \\
6 \text { months }\end{array}$ & 48 & $\begin{array}{l}\text { Higher } \mathrm{Hb} \text { at lower EPO doses for HF-HD } \\
\text { Higher MM removal }\end{array}$ \\
\hline Bonomini et al. [44] & $\begin{array}{l}\text { Prospective, randomized crossover; HF-HD versus } \\
\text { OL-HDF }\end{array}$ & 8 & $\begin{array}{l}\text { Enhanced elimination of uremic toxins that promote } \\
\text { exposure of phosphatidylserine on RBC to prolong life } \\
\text { span }\end{array}$ \\
\hline Hsu et al. [59] & $\begin{array}{l}\text { Prospective crossover, switch from conventional to } \\
\text { ultrapure dialysate, } 12 \text { months }\end{array}$ & 34 & $\begin{array}{l}\text { Reduced inflammation (CRP) with endotoxin-free } \\
\text { dialysate } \\
\text { Reduced EPO doses } \\
\text { Improved iron utilization }\end{array}$ \\
\hline Lin et al. [27] & Prospective, switch from conventional HD to OL-HDF & 92 & $\begin{array}{l}\text { Reduced EPO resistance and dose; higher Hct } \\
\text { Improved iron utilization with OL-HDF }\end{array}$ \\
\hline Bonforte et al. [26] & $\begin{array}{l}\text { 9-month observation after switch from LF-HD to } \\
\text { OL-HDF }\end{array}$ & 32 & Higher $\mathrm{Hb}$ and reduced EPO doses \\
\hline Movilli et al. [65] & Examine relationship between Kt/V and EPO dose & 65 & Reduction in EPO doses at higher dialysis dose \\
\hline Maduell et al. [28] & $\begin{array}{l}\text { Switch from conventional HD to OL-HDF over 1-year } \\
\text { period }\end{array}$ & 37 & $\begin{array}{l}\text { Increase in } \mathrm{Hb} \text { and } \mathrm{Hct} \\
\text { Decrease in EPO doses } \\
\text { Higher MM removal }\end{array}$ \\
\hline
\end{tabular}

intestinal calcium absorption, causes elevated PTH in CKD. Restriction of hyperphosphatemia may thus provide further benefits in terms of anemia control in addition to those recognized for mineral bone disease. It is to be noted that of all the uremic retention solutes regarded as potential uremic toxins, phosphate is perhaps the most widely acknowledged substance to express in vivo toxic- ity. Enhanced reduction in phosphate levels during HD, together with phosphate binders, may contribute to anemia control by restricting increase in PTH levels (fig. 1). The effectiveness of OL-HDF in reducing hyperphosphatemia has been demonstrated in a number of studies resulting in significantly higher removal of phosphate than HF-HD [33-35]. 
Increase in Iron Availability during Dialysis

Background: Interference of Iron Metabolism in

CKD Promotes Anemia

Adequate erythropoietic function requires, in addition to EPO, about $20-30 \mathrm{mg} /$ day iron provided mainly through internal turnover as well as by absorption of dietary iron from the duodenum. Absence of iron deprives the basic building block molecule of RBC in the latter stages of the erythropoietic process. In CKD, multiple forms of interference of all phases of iron metabolism (absorption and utilization) as well as inhibition of iron release from the reticuloendothelial system occur [36]. Inadequate iron uptake, together with chronic blood loss (bleeding events, accidental losses, excessive blood drawn for laboratory tests, blood retained within the dialysis circuit after termination of each HD session), means that up to $3 \mathrm{~g}$ of iron may be lost annually in an average $\mathrm{HD}$ patient [37].

Hepcidin, a 25 -amino acid acute-phase reacting protein produced and secreted by the liver but excreted by the kidneys, is a key mediator of systemic iron homeostasis and responsible for the disturbed iron metabolism in anemia of CKD [38]. While hepcidin may not itself exert toxicity, it is nevertheless listed by the European Uremic Toxin Work Group as an MM whose removal is likely to be beneficial [3]. Increased expression of hepcidin decreases intestinal iron absorption and inhibits iron release from reticuloendothelial and hepatocyte stores leading to iron-deficient anemia of CKD [39] (fig. 1). With a molecular weight of $2,789 \mathrm{Da}$, hepcidin would be amenable to removal by treatment modalities using highflux membranes. Data regarding elimination of hepcidin by HD are scarce, but HF-HD (unlike LF-HD) predicts substantial decrease in serum hepcidin levels [40]. Anemia correction observed for convective treatment modalities (e.g. OL-HDF) could, in part, be attributed to a more efficient reduction in hepcidin together with reduced inflammation induced by OL-HDF [41].

\section{Impact of Dialysis on Erythrocyte Life Span}

\section{Background: Reduced Life Span and Accelerated Destruction of Circulating RBC in CKD}

In $\mathrm{CKD}$, erythrocytes, once produced and released into the circulation from bone marrow, exhibit a distinct shorter life span and an increased propensity to destruction [42]. Increased erythrocyte deformability and abnormalities of plasma membrane symmetry and cytoskeleton affect RBC life span and promote phagocytosis
[21]. Functional changes and destruction of the membrane are accelerated by the uremic environment, inflammation and oxidative stress [42]. Metabolic derangements associated with uremia and uremic toxicity thus affect both the production and survival of RBC [21] (fig. 1).

Solutes retained during uremia cause increased exposure of phosphatidylserine (PS) from the inner to the outer membrane leaflet of RBC [21]. The externalization of PS represents a signal for recognition by macrophages and subsequent elimination of deformed RBC from the circulation; furthermore, adhesion of these abnormal $\mathrm{RBC}$ to vascular endothelium accelerates pathogenesis of vascular disease commonly encountered in CKD [43]. Uremic plasma and ultrafiltrate taken from patients treated with OL-HDF has been shown to significantly reduce PS expression on normal erythrocytes compared to those treated with HF-HD with the same dialysis membrane [44]. The results were attributed to the increased elimination of a putative uremic compound that is lipophilic, probably associated with proteins and having a molecular weight of between 10 and $10.8 \mathrm{kDa}$. Convective therapies may thus retard the uremia-induced consumption and reduced life span of RBC.

\section{Impact of Dialysis on Amplification of Anemia by Inflammation}

\section{Background: Chronic Inflammation Augments \\ Anemia of CKD}

Systemic inflammation is highly prevalent in all CKD patients and believed to be a major contributor to morbidity and mortality in this patient population [45]. Underlying inflammation, together with oxidative stress, is closely linked to endothelial dysfunction, vascular injury and atherosclerosis, explaining the high prevalence of cardiovascular disease in patients with CKD $[1,2]$. Chronic inflammation and acute-phase response are believed to interact at several levels to impair erythropoiesis, accelerate destruction of erythrocytes and/or blunt the increase in production of EPO in response to hypoxia [46]. Cytokines, including IL-1, IL- 6 and TNF- $\alpha$, suppress and affect precursor cells at different stages of erythropoiesis [47]. Inflammation may also impair iron availability for erythropoiesis: HD patients with high CRP levels ( $>8 \mathrm{mg} / \mathrm{l})$ have lower iron absorption than patients with lower CRP levels [48]. Significantly, inflammation regulates hepcidin expression and production in response to liver iron levels, hypoxia and anemia [49] (fig. 1). Molecular mechanisms 
by which inflammation (including cytokines and bacterial lipopolysaccharide, LPS) induces hepatic hepcidin expression have been elucidated and reviewed by Babbit and Lin [39]. Modulation of iron metabolism by cytokines during inflammation leads to functional iron deficiency or to increased ferritin and decreased transferrin production, shunting iron to the reticuloendothelial storage pool instead of delivery to erythrocyte precursors [50]. Mechanisms involving inflammation that cause shortened half-life of erythrocytes and iron deficiency have also been described [51]. Inflammation thus acts in concert with uremic toxicity and hepcidin to exacerbate anemia at different stages (fig. 1). Moreover, some uremic toxins express potent proinflammatory and oxidative activity. For instance, substances like p-cresylsulfate (a metabolite of p-cresol) and those arising from protein glycation and oxidation (e.g. advanced glycation end products) are potent in promoting inflammation, thereby aggravating anemia during $\mathrm{CKD}[52,53]$.

\section{Dialysis Strategies That Curb the Effects of \\ Inflammation}

Dialysis modalities that suppress the effects of mediators of inflammation (together with removal of putative inhibitors of erythropoiesis) would be expected to curtail decreased erythropoiesis in CKD. Carracedo et al. [54] demonstrated a marked reduction in the proinflammatory state by OL-HDF and high-efficiency convective therapies; a significantly lower number of CD14+CD16+expressing peripheral monocytes as well as decreased TNF- $\alpha$ and IL- 6 production were observed in 31 patients treated with OL-HDF compared to those treated with HF-HD. Reduced inflammation by OL-HDF is attributed to the combined effects of efficient removal of proinflammatory substances and inhibitors of erythropoiesis, and to the usage of microbiologically pure dialysis fluids [55].

In all forms of HD therapies, particularly high-efficiency convective modalities requiring large volumes of substitution fluid, a major potential source of inflammation and oxidative stress is dialysis fluid contaminated with microbiological impurities [56]. LPS, generated during bacterial growth and lysis, are easily able to traverse HF dialysis membranes, entering the bloodstream to cause persistent, low-grade inflammation by activating mononuclear cells to release proinflammatory cytokines and free oxygen radicals [57]. Contemporary dialysis technology, however, allows the cost-effective 'online' production of large amounts of microbiologically pure fluids to ensure that the patient is protected from additional inflammatory and oxidative stress stimuli. Hence, application of ultrapure water (obtained with endotoxinretaining filter systems) is strongly recommended for all forms of HD therapy [58]. A number of studies have demonstrated that use of ultrapure dialysis fluids with OLHDF therapy has beneficial effects for erythropoiesis and anemia treatment; reduced EPO doses, improved iron utilization and extended RBC life span have been attributed to the usage of ultrapure dialysis fluids $[59,60]$.

Combating the detrimental effects of uremic toxicity, inflammation, oxidative stress and endothelial dysfunction appears to be fundamental for correction of anemia of CKD. It is important to emphasize the synergy between these underlying states; in addressing anemia of $\mathrm{CKD}$, any approach that restricts progression of the underlying conditions that aggravate the condition should be utilized. For example, certain pharmaceutical preparations may, themselves, induce higher inflammatory and oxidative stress responses. Some forms of iron therapy may trigger unfavorable effects on immune function with clinical implications including affecting the degree of anemia [61]. Toblli et al. [62] observed deleterious hemodynamic, inflammatory and oxidative stress responses with certain i.v. iron preparations in an animal model. Better control of anemia may further be achieved by combination of preventive measures (involving both dialysis and pharmaceutical interventions) that restrict mechanisms known to amplify anemia.

\section{Dialysis Strategies That Affect Anemia Therapy}

Hyporesponsiveness to EPO therapy is a common complication during management of anemia in HD patients [63]. Mechanisms involving iron deficiency or utilization, deficiency of other nutrients, infections and inflammation, uremic toxins and inadequate dialysis are some of the main pathways believed to contribute to episodes of such resistance. An inadequate response to EPO therapy is defined as failure to achieve or maintain target $\mathrm{Hb} / \mathrm{Hct}$ levels in the presence of adequate iron stores at specified intravenous or subcutaneous doses of EPO within a certain period of time [64]. Dialysis therapy not only impacts the processes of bone marrow erythropoiesis, circulating erythrocytes, uremic toxicity and inflammation, but anemia therapy itself - particularly EPO resistance [63].

Dialysis Strategies That Increase EPO Responsiveness

There is evidence showing that the intensity or adequacy of dialysis is a contributing factor towards the op- 
Fig. 2. Mechanisms by which OL-HDF could provide benefits in terms of anemia control. Published data show that OLHDF favorably impacts anemia of CKD by not only removing putative inhibitors that suppress erythropoiesis, reducing red cell destruction and increasing iron availability, but also by restricting underlying conditions affecting anemia therapy.

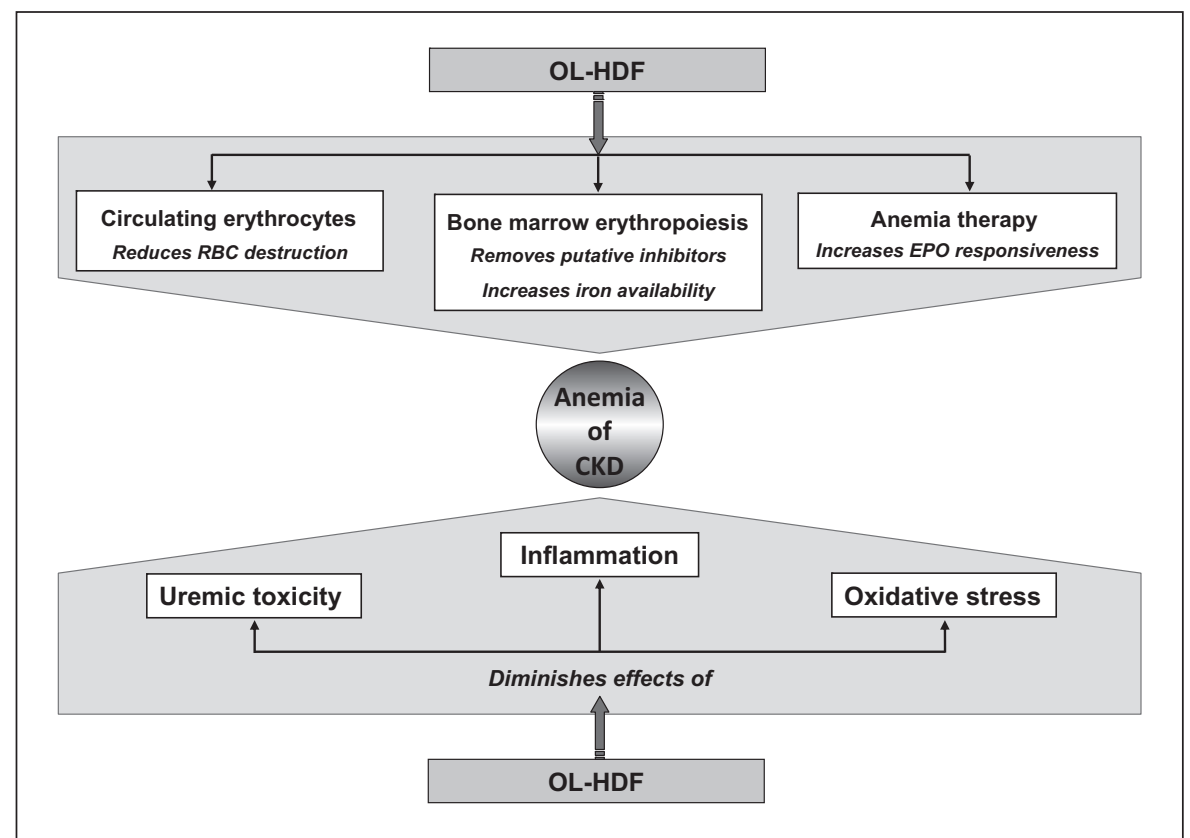

timization of exogenous EPO responsiveness [25, 51, 65]. Switching patients from conventional HD to OL-HDF was associated with a reduction in EPO dose to reach a higher mean Hct level $[27,28]$. In a study in which patients on conventional HDF were changed to OL-HDF, an EPO dose reduction of $16 \%$ EPO was observed after the switch to OL-HDF, with significant increase in $\mathrm{Hb}$ and Hct levels during the OL-HDF period [26]. In another prospective crossover clinical evaluation, patients on conventional LF-HD were randomized to HF-HD or OLHDF for 24 months and then switched to the alternative treatment for a further 24 months; both high-flux modes of RRT significantly improved the hematopoietic response to $\mathrm{rHuEPO}$ in parallel with significant decreases in low-grade microinflammation [66]. The dose of EPO necessary to maintain target $\mathrm{Hb}$ levels was 24 and $27 \%$ less in patients on HF-HD and OL-HDF, respectively, than in patients on conventional LF-HD. OL-HDF, by virtue of its ability to enhance removal of larger uremic toxins that suppress erythropoiesis and cause less inflammation, thus appears to have a role in decreasing EPO resistance and improved iron utilization (fig. 2). However, not all studies have been able to observe a decrease in EPO resistance for HDF compared to HF-HD [10].

EPO resistance is associated with survival in HD patients: a greater EPO responsiveness has been shown to be associated with improved survival [67]. Recent results from the RISCAVID Study (prospective, 36-month observational study involving 753 patients in Tuscany, Italy) showed further that both resistance to ESA as well as HD (compared to HDF) increased the relative risk of all-cause mortality and fatal/non-fatal cardiovascular events [68]. Significantly, in showing a direct correlation between IL-6 (rather than CRP) levels and increased ESA requirements/resistance, the authors reinforce the involvement of mechanisms such as inflammation that interfere with the actions of ESA. Although the RISCAVID Study is not an RCT, the evidence from the large cohort of patients in HD units nonetheless indicates that better dialysis therapy that is both more efficient (OL-HDF) and curtails inflammation could improve EPO responses.

\section{Removal of Inhibitors of Erythropoiesis, Repletion of Iron Stores, Reduced Inflammation and Response to EPO by OL-HDF: Results of a Prospective RCT}

A recently published prospective, multicenter randomized crossover study on the long-term effects of highefficiency OL-HDF confirms the potential impact of OLHDF on the aforementioned mechanisms (pertaining to anemia control) described in this communication [69]. Significantly enhanced removal of diverse markers of uremic toxicity (including diminution of PTH and Caphosphate product), repletion of iron stores and reduced 
inflammation all corresponded with a reduction in EPO doses with OL-HDF compared to LF-HD at comparable $\mathrm{Hb}$ levels for the two treatment modalities. The authors reiterate the benefits and ramifications of high-efficiency convective treatments on a host of uremia-related derangements, including anemia of $\mathrm{CKD}$, which may contribute to improved patient survival.

\section{Conclusions: Can Dialysis Strategies Enable Better Management of Anemia?}

The scientific reasoning and efficacy of OL-HDF are rarely disputed: it is acknowledged to be the most efficient form of extracorporeal RRT for the elimination of most small and large uremic retention solutes that accumulate in uremia. Abundant publications attest to its clinical validation, and there appear to be no safety concerns or reports of OL-HDF being detrimental. Patients vouch for improved well-being, suffering fewer complications and are reluctant to revert to less efficient treatment options. Considerable available evidence based on observational, epidemiological or data from small prospective randomized crossover trials indicates improved patient outcomes with OL-HDF $[1,10]$. Yet, while recognizing these advantages, some nephrologists nevertheless hesitate to prescribe OL-HDF, citing the need for more definitive evidence in the form of prospective, well-powered, large-scale, multi-center RCT showing a survival advantage for OL-HDF.

Until such conclusive data become available, the vast majority of the HD patients may, arguably, be deprived of benefits of a therapy that has been associated with better quality of life and may well prolong patient life span. The fact remains that OL-HDF, far from being an experimental treatment, is a therapeutic intervention in routine clinical use in several countries over extended periods, in many centers exceeding a decade. Almost $12 \%$ of the dialysis population worldwide is treated with this therapy, with an upward trend. The paradox with other dialysis practices that have not been supported by evidence from RCT cannot be ignored: for example, both the duration and frequency of current dialysis treatments have not been backed by prospective, large-scale RCT. Longer treatment times $(>3 \mathrm{~h}$ or even $>4 \mathrm{~h}$ and extended nocturnal $8 \mathrm{~h}$ ) or treatments delivered more than thrice weekly are being widely used and advocated, with cost considerations rather than lack of evidence from RCT being the only deterrent. Despite the quest for the holy grail of exclusively evidence-based interventions, the annals of medicine are replete with examples of highly successful therapeutic interventions and practices carried out routinely without the seal of RCT [70].

Application of convective therapies is a clinical reality, and it would perhaps be more pertinent to examine the reasons for its continued usage and success. Most published appraisals of OL-HDF only review or assess existing evidence, listing the diverse benefits that have been reported for the therapy. In this paper, we have analyzed the various biochemical mechanisms by which OL-HDF may construe its purported advantages. We have selected the example of anemia for two reasons: firstly, there are several reports of anemia correction and EPO dose reduction ascribed to HF-HD and OL-HDF. Secondly, anemia remains a major issue for many HD patients despite widespread usage of costly ESA and iron supplements to suppress the condition; anemia management strategies are under considerable scrutiny and are being reevaluated [71-73].

In this communication, we have examined the potential role of dialysis strategies, beyond and including EPOiron therapies, towards improved anemia control in HD patients. We believe revised strategies that take into account the impact of dialysis procedures on pathways and factors involved in anemia are needed. Dialysis therapy impacts three key aspects of anemia of CKD: processes of erythropoiesis, its product (erythrocytes) and anemia therapy itself. For each of the three categories, we have provided examples of published evidence showing how advanced dialysis treatment modalities may positively modulate anemia of CKD. We have further shown that dialysis approaches that are able to restrict the combined effects of uremic toxicity and inflammation (and oxidative stress) would better complement conventional pharmaceutical therapy interventions used for anemia management.

While the efficacy of OL-HDF in combating the effects of uremic toxicity remains the overriding consideration for its prescription, a growing clinical experience with this treatment modality is beginning to reveal its versatility. The evidence so far indicates that OL-HDF may alleviate the effects of a number of conditions afflicting dialysis patients, including anemia. Dialysis therapy, performed as efficiently as possible, may have the potential to provide end-stage CKD patients significant benefits beyond the life-saving function of removal of accumulated waste products and excess fluid. Further optimization and integration of the dialysis and pharmaceutical interventions may be more effective in overcoming some of the present shortcomings of adequate ane- 
mia control in HD patients whose treatment presently relies almost entirely on costly exogenous ESA/iron therapy [74]. There is evidence to indicate that the judicious selection of the dialysis treatment modality, or of components of the procedure, has the potential to improve the anemic condition of CKD patients. In the search for more effective, safer and less expensive approaches to anemia management, the effects of the dialysis proce- dure should also be considered in conjunction with pharmaceutical therapy interventions that are needed to correct anemia of CKD.

\section{Acknowledgements}

We would like to thank Dr. H. Feidhlim Woods for his guidance.

\section{References}

$\checkmark 1$ Himmelfarb J, Ikizler TA: Hemodialysis. N $>14$ Locatelli F, Manzoni C, Cavalli A, Di Filippo Engl J Med 2010;363:1833-1845.

$\checkmark 2$ Meyer TW, Hostetter TH: Uremia. N Engl J Med 2007;357:1316-1325.

$\checkmark 3$ Vanholder R, van Laecke S, Glorieux G What is new in uremic toxicity? Pediatr Nephrol 2008;23:1211-1221.

4 Bowry SK, Kuchinke-Kiehn U, Ronco C: The cardiovascular burden of the dialysis patient: the impact of dialysis technology. Contrib Nephrol 2005;149:230-239.

$\checkmark 5$ Cavalli A, Del Vecchio L, Manzoni C, Locatelli F: Hemodialysis: yesterday, today and tomorrow. Minerva Urol Nefrol 2010;62:111.

6 6 Locatelli F, Martin-Malo A, Hannedouche T, et al: Effect of membrane permeability on survival of hemodialysis patients. J Am Soc Nephrol 2009;20:645-654.

$\checkmark 7$ Ronco C: Hemodiafiltration: evolution of technique towards better dialysis care. Contrib Nephrol 2011;168:19-27.

$\checkmark 8$ Canaud B, Chenine L, Henriet D, Leray H: Online hemodiafiltration: a multipurpose therapy for improving quality of renal replacement therapy. Contrib Nephrol 2008; 161:191-198.

$\checkmark 9$ Ledebo I: Convective dialysis therapies, current status and perspective. Ther Apher Dial 2005;9:223-227.

$\checkmark 10$ van der Weerd NC, Penne EL, van den Dorpel MA, et al: Haemodiafiltration: promise for the future? Nephrol Dial Transplant 2008;23:438-443.

-11 Canaud B, Bragg-Gresham JL, Marshall M, et al: Mortality risk for patients receiving hemodiafiltration versus hemodialysis: European results from the DOPPS. Kidney Int 2006;69:2087-2093.

-12 Jirka T, Cesare S, Di Benedetto A, et al: Mortality risk for patients receiving hemodiafiltration versus hemodialysis: European results from the DOPPS. Kidney Int 2006;70: 1524.

13 Penne EL, Blankestijn PJ, Bots ML, et al: Resolving controversies regarding hemodiafiltration versus hemodialysis: the Dutch Convective Transport Study. Semin Dial 2005;18: 47-51.
S: Can convective therapies improve dialysis outcomes? Curr Opin Nephrol Hypertens 2009; 18:476-480.

15 Panichi V, Tetta C: The biological response to online hemodiafiltration. Contrib Nephrol 2007; 158:194-200.

16 Lankhorst CE, Wish JB: Anemia in renal disease: diagnosis and management. Blood Rev 2010;24:39-47.

17 Wish JB: The economic realities of erythropoiesis-stimulating agent therapy in kidney disease. Kidney Int 2006;70:S21-S25.

$\checkmark 18$ Kovesdy CP, Kantar-Zadeh K: Emerging challenges of anemia management in CKD. Adv Chronic Kidney Dis 2009;16:74-75.

19 Coyne DW: It's time to compare anemia management strategies in hemodialysis. Clin J Am Soc Nephrol 2010;5:740-742.

20 Ohno Y, Rege AB, Fisher JB, Barona J: Inhibitors of erythroid colony forming cells CFU-E and BFU-E in sera of azotemic patients with anemia of renal disease. J Lab Clin Med 1978;92:916-923.

21 Bonomini M, Sirolli V: Uremic toxicity and anemia. J Nephrol 2003;16:21-28.

22 Nolph KD: Comparison of continuous ambulatory peritoneal dialysis and hemodialysis. Kidney Int 1988;33:S123-S131.

23 Mcdougall I: Role of uremic toxins in exacerbating anemia in renal failure. Kidney Int 2001;58:67-72.

24 Yamada S, Kataoka H, Kobayashi H, Ono T, Minakuchi J, Kawano Y: Identification of an erythropoietic inhibitor from the dialysate collected in hemodialysis with PMMA membrane (BK-F). Contrib Nephrol 1999;125: 159-172.

25 Ayli D, Ayli M, Azak A, et al: The effect of high-flux hemodialysis on renal anemia. J Nephrol 2004;17:701-706.

-26 Bonforte G, Grillo P, Zerbi S, Surian M: Improvement of anemia in hemodialysis patients treated by hemodiafiltration with high-volume online prepared fluid. Blood Purif 2002;20:357-363.

27 Lin CL, Huang CC, Yu CC, et al: Improved iron utilisation and erythropoietin resistance by online haemodiafiltration. Blood Purif 2002;20:349-356.
28 Maduell F, del Pozo C, Garcia H, Sanchez L, Hdez-Jaras J, Albero MD, Calvo C, Torregrosa I, Navarro V: Change from conventional haemodiafiltration to on-line haemodiafiltration. Nephrol Dial Transplant 1999;14: 1202-1207.

29 Galli F, Beninati S, Benedetti S, et al: Polymeric protein-polyamine conjugates: a new class of uremic toxins affecting erythropoiesis. Kidney Int 2001;59:S73-S76.

30 Lutton JD, Solangi KB, Ibraham NG, Goodman AI, Levere RD: Inhibition of erythropoiesis in chronic renal failure. The role of parathyroid hormone. Am J Kidney Dis 1984;5:380-384.

-31 Hörl WH: The clinical consequences of secondary hyperparathyroidism: focus on clinical outcomes. Nephrol Dial Transplant 2004;19:V2-V8.

32 Almeras C, Argiles A: The general picture of uremia. Semin Dial 2009;22:329-333.

33 Vaslaki L, Major L, Berta K, et al: On-line haemodiafiltration versus haemodialysis: stable haematocrit with less erythropoietin and improvement of other relevant blood parameters. Blood Purif 2006;24:163-173.

34 Zehnder C, Gutzwiller J-P, Renggli K: Hemodiafiltration - a new treatment option for hyperphosphatemia in hemodialysis patients. Clin Nephrol 1999;52:152-159.

35 Lornroy W, De Meester J, Because I, Billiouw J-M, Van Malderen PA, Van Pottelberge M: Impact of convective flow on phosphorous removal in maintenance hemodialysis patients. J Renal Nutr 2006;16:47-53.

36 Garneata L: Intravenous iron, inflammation, and oxidative stress: is iron a friend or an enemy of uremic patients? J Renal Nutr 2008;18:40-45.

>37 Kalantar-Zadeh, Streja E, Miller JE, Nissenson AR: Intravenous versus erythropoiesisstimulating agents: friends or foes in treating chronic kidney disease anemia? Adv Chronic Kid Dis 2009;16:143-151.

38 Ganz T: Hepcidin and its role in regulating systemic iron metabolism. Hematology Am Soc Hematol Educ Program 2006; 1:29-35. 
39 Babitt JL, Lin HY: Molecular mechanisms of hepcidin regulation: implications for the anemia of CKD. Am J Kidney Dis 2010;55: 726-741.

40 Zaritsky J, Young B, Gales B, et al: Reduction of serum hepcidin by hemodialysis in pediatric and adult patients. Clin J Am Soc Nephrol 2010;5:1010-1014.

41 Malyszko J, Malyszko JS, Kozminski P, Mysliwiec M: Type of renal replacement therapy and residual renal function may affect prohepcidin and hepcidin. Renal Fail 2009;31:876-883.

-42 Kruse A, Uehlinger DE, Gotch F, Kotanko P, Levin NW: Red blood cell lifespan, erythropoiesis and haemoglobin control. Contrib Nephrol 2008;161:247-254.

43 Pandolfi A, Di Pietro N, Sirolli V, et al: Mechanisms of uremic erythrocyte-induced adhesion of human monocytes to cultured endothelial cells. J Cell Physiol 2007;213:699709.

44 Bonomini M, Ballone E, Di Stante S, et al: Removal of uraemic plasma factor(s) using different dialysis modalities reduces phosphatidylserine exposure in red blood cells. Nephrol Dial Transplant 2004;19:68-74.

45 Stenvinkel P: New insights on inflammation in chronic kidney disease-genetic and nongenetic factors. Nephrol Ther 2006;2:111119 .

46 Chawla LS, Krishnan M: Causes and consequences of inflammation on anemia management in haemodialysis patients. Hemodial Int 2009;13:222-234.

-47 Allen DA, Breen C, Yaqoob MM, Macdougall IC: Inhibition of CFU-E colony formation in uremic patients with inflammatory disease: role of IFN-gamma and IL-1. J Invest Med 1999;47:204-211.

$\checkmark 48$ Kooistra MP, Niemantsverdriet EC, van Es A, Mol-Beerman NM, Struyvenberg A, Marx JJ: Iron absorption in erythropoietin-treated haemodialysis patients: effects of iron availability, inflammation and aluminium. Nephrol Dial Transplant 1998;13:82-88.

$\checkmark 49$ Eleftheriadis T, Liaopoulos V, Antoniadi G, Kartsios C, Stefanidis I: The role of hepcidin in iron homeostasis and anemia in hemodialysis patients. Semin Dial 2009;22:70-77.

50 De Francisco ALM, Stenvinkel P, Vaulot S: Inflammation and its impact on anemia in chronic kidney disease: from haemoglobin variability to hyporesponsiveness. Nephrol Dial Transplant Plus 2009;2:i18-i26.
1 Nangaku M, Eckardt KU: Pathogenesis of renal anemia. Semin Nephrol 2006;26:261268.

-52 Schepers E, Meert N, Glorieux G, Goeman J, Van der Eycken J, Vanholder R: P-cresylsulfate, the main in vivo metabolite of p-cresol, activates leucocyte free radical production. Nephrol Dial Transplant 2007;22:592-596.

53 Piroddi M, Depunzio I, Calabrese V, et al: Oxidatively-modified and glycated proteins as candidate pro-inflammatory toxins in uremia and dialysis patients. Amino Acids 2007;32:573-592.

54 Carracedo J, Merino A, Nogueras S, et al: On-line hemodiafiltration reduces the proinflammatory CD14+CD16+ monocyte-derived dendritic cells: a prospective, crossover study. J Am Soc Nephrol 2006;17:23152321.

55 Panichi V, Rizza GM, Paoletti S, et al: Chronic inflammation and mortality in haemodialysis: effect of different renal replacement therapies. Results from the RISCAVID study. Nephrol Dial Transplant 2008;23: 2337-2343.

56 Schindler R: Inflammation and dialysate quality. Hemodial Int 2006;10:S56-S59.

57 Lonnemann G: Chronic inflammation in hemodialysis: the role of contaminated dialysate. Blood Purif 2000;18:214-223.

58 European Best Practice Guideline for Haemodialysis Part 1. IV.1 Water treatment system. Nephrol Dial Transplant 2002;17:4562 .

59 Hsu P-Y, Lin C-L, Yu C-C, et al: Ultrapure dialysate improves iron utilisation and erythropoietin response in chronic hemodialysis patients - a prospective cross-over study. J Nephrol 2004;17:693-700.

60 Go I, Takemoto Y, Tsuchida K, Sugimura K, Nakatani T: The effect of ultrapure dialysate on improving renal anemia. Osaka City Med J 2007;53:17-23.

61 Weiss G, Meusburger E, Radacher G, Garimorth K, Neyer U, Mayer G: Effect of iron treatment on circulating cytokine levels in ESRD patients receiving recombinant erythropoietin. Kidney Int 2003;64:572-578.

62 Toblli JE, Cao G, Oliveri L, Angerosa M: Comparison of the renal, cardiovascular and hepatic toxicity data of original intravenous compounds. Nephrol Dial Transplant 2010; 25:3631-3640.

63 Kanbay M, Perazella MA, Kasapoglu B, Koroglu M, Vovic A: Erythropoiesis stimulatory agent-resistant anemia in dialysis patients: review of causes and management. Blood Purif 2010;29:1-12
64 Priyadarshi A, Shapiro JI: Erythropoietin resistance in the treatment of anemia of chronic renal failure. Semin Dial 2006;19:273-278.

65 Movilli E, Cancarini GC, Zani R, Camerini C, Sandrini M, Maiorca R: Adequacy of dialysis reduces the doses of recombinant erythropoietin independently from the use of biocompatible membranes in haemodialysis patients. Nephrol Dial Transplant 2001; 16:111-114.

66 Schiffl H: Prospective randomized crossover long-term comparison of online haemodiafiltration and ultrapure high-flux haemodialysis. Eur J Med Res 2007;12:2633.

67 Kilpatrick RD, Critchlow CW, Fishbane S, et al: Greater epoetin alfa responsiveness is associated with improved survival in hemodialysis patients. Clin J Am Soc Nephrol 2008; 3:1077-1083

68 Panichi V, Rosati A, Bigazzi R, et al: Anemia and resistance to erythropoiesis-stimulating agents as prognostic factors in haemodialysis patients: results from the RISCAVID Study. Nephrol Dial Transplant 2011, Epub ahead of print.

69 Pedrini LA, De Cristofaro V, Comelli M, et al: Long-term effects of high-efficiency online haemodiafiltration on uremic toxicity: a multi-centre prospective randomised study. Nephrol Dial Transplant 2011, Epub ahead of print.

70 Smith GCS, Pell JP: Parachute use to prevent death and major trauma related to gravitational challenge: systematic review of randomised controlled trials. BMJ 2003;327: 1459-1461.

71 Unger EF, Thomson AM, Blank MJ, Temple R: Erythropoiesis-stimulating agents - time for a reevaluation. N Engl J Med 2010;21: 189-192.

72 Spiegel DM, Chertow GM: Lost without directions: lessons from the anemia debate and the Drive Study. Clin J Am Soc Nephrol 2009;4:1009-1010.

73 Locatelli F, Aljama P, Canaud B, et al: Target haemoglobin to aim for with erythropoiesisstimulating agents: a position statement by ERBP following publications of the Trial to Reduce Cardiovascular Events with Aranesp Therapy (TREAT) Study. Nephrol Dial Transplant 2010;25:2846-2850.

74 Kotanko P, Levin NW: How can erythropoietin requirements be reduced in dialysis patients? Sem Dial 2006;19:363-364. 